\title{
Dielectric Properties of Oligomers. VIII. The Dielectric Properties of Model Molecules for Poly(vinyl acetate)
}

\author{
Eiji IKADA, Kazunari SHounAKA, and Michio ASHIDA \\ Faculty of Engineering, Kobe University, \\ Rokkodai-cho Nada-ku, Kobe 657, Japan.
}

(Received June 21, 1980)

\begin{abstract}
The dielectric properties of 2,4,6-heptanetriol triacetate, a model substance for poly(vinyl acetate), were studied to clarify significant roles played by the molecular structure in the $\alpha$-relaxation behavior of polymers. At room temperature, this trimer of vinyl acetate is a viscous mixture of three stereoisomers, i.e., syndio-, iso-, and hetero-tactic 2,4,6-heptanetriol triacetate. Dielectric measurements for each isomer and for the mixture were carried out in a frequency range from $23 \mathrm{~Hz}$ to $3 \mathrm{MHz}$ in a pure liquid state. The dipole moments in a carbon tetrachloride solution were also measured. The static dielectric constant was largest for the syndiotactic compound and smallest for the isotactic compound. In the above frequency range, the syndiotatic and isotatic isomers showed dielectric dispersions due to rotation of the molecule as a whole and the relaxation parameters were calculated by means of the asymmetric Havriliak-Negami empirical equation. It was found that the distribution of relaxation times for the isomers is almost the same as that for poly(vinyl acetate). It was concluded that the width and asymmetry of the distribution, at least in the case of the vinyl acetate trimer, can not be attributed to the fact that this trimer is a mixture of the three stereoisomers.

KEY WORDS Dielectric Relaxation / Model Compound / Poly(vinyl acetate) / Stereoisomer / Distribution of Relaxation Times / Havriliak-Negami Type Relaxation /
\end{abstract}

Mead and Fuoss ${ }^{1}$ reported on the dielectric relaxation of poly(vinyl acetate) in the rubbery state and later Ishida et al. $^{2}$ made a more complete study of this matter. The shape of the arc reported for the Cole-Cole plane resembles well the one observed for hydrogen-bonded liquid glycols. ${ }^{3}$ This apparent resemblance found in the two substances of quite different molecular structure, was of interest, although there was no theoretical explanation to account for this similarity.

In order to find the common relaxational properties responsible for the resemblance between a liquid of a small molecule and a rubbery polymer, the dielectric relaxations of the following acetate chainmolecules were studied:

Glycerol Triacetate and 1,2,6-Hexanetriol Triacetate. $^{4}$

These two triacetates have no vinyl-type structure and their molecular weights are much smaller than that of poly(vinyl acetate). The dielectric measurement shows that the arcs of their complex dielectric constants, determined from the distribution of relaxation times, are almost the same as that of poly(vinyl acetate) in spite of the very different relaxation mechanism. This led to the conclusion that the acetate side-group is associated with the distribution of relaxation times and that the distribution has nothing to do with the chain-length.

Low-Molecular-Weight Poly(vinyl acetate) prepared by the Vinyl Polymerization. ., $^{-}$

All oligomers in this study showed relaxation time distribution wider than that for poly(vinyl acetate). This seems unreasonable because it is impossible for properties in a polymeric system to be simpler than the corresponding properties in an oligomeric systems.

The oligomers in this study (the average degree of polymerization $=3.0-4.7^{6}$ ) had the polar end- 
groups (-OC( $\left(\mathrm{CH}_{3}\right)_{3},-\mathrm{Cl},-\mathrm{Cl}_{3}$, etc.). As far as we know, these polar chain-ends, produced via transfer of decomposed initiators and solvent radicals to the chains, are inevitable as well as the moelcularweight distribution. The additional relaxation of the polar end-groups and the molecular-weight distribution broaden the relaxation time distributions of these oligomers. The principal relaxation in the oligomers is due to rotation of the entire molecule. The coexistence of molecules of various size enhances the relaxation time distribution. The principal relaxation in polymers ( $\alpha$-relaxation) is due to segmental motion. The distribution of molecular weight has almost no effect on the distribution of relaxation time because the segmental unit in the main chain is much shorter than the length of the chain. Therefore, we tried to obtain a model substance having dielectric properties that would confirm the conclusions of studies I and II.

2,4,6-Heptanetriol triacetate, which has neither a polar end-group introduced during the polymerization process nor a distribution of molecular weight, will prove useful in checking a wide distribution of relaxation times for oligomers as indicated by study II. In addition to the distribution of molecular weight, another important diversity in polymers results from the rotational and geometrical isomerism of repeating units. These diversities are perhaps associated with the property characteristics of polymeric systems, such as the wide distribution of relaxation times in the rheological properties.

From this standpoint, it is worthwhile to study the properties of oligomers which are free from the complexities of polymeric systems. A study on a simplified system will provide basic information needed for investigating polymeric materials.

The recent availability of a distillator of high theoretical plates has made it possible to separate an organic substance consisting of isomers with nearly equal boiling points into pure isomers.

2,4,6-Heptanetriol triacetate has the three different stereoisomers illustrated below.

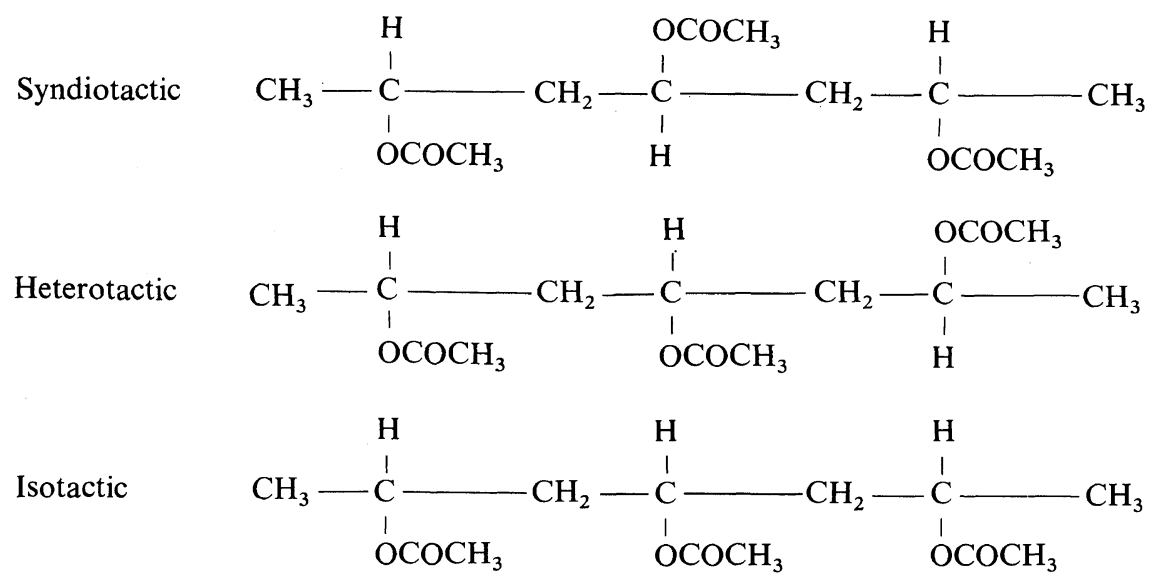

The structural analysis of polymers by nuclear magnetic resonance (NMR) requires accurate information regarding the relationships between a fine structure in a chain and the chemical shifts in absorption peaks. Several studies on the fine structures of a trimer and the related molecules have been reported..$^{7,8}$ Generally, even a chain oligomer has many isomers since it can take on any number of spatial arrangements. It is of interest and useful to compare the properties of isomers and investigate the contribution of structural diversities toward the various properties of oligomers.

The purpose of this investigation is to determine whether the asymmetric relaxation time distributions in $\alpha$-relaxation are associated with an isomeric mixture and the distribution of molecular weight.

\section{EXPERIMENTAL}

\section{Preparation of 2,4,6-Heptanetriol Triacetate}

2,4,6-Heptanetriol was prepared by the method 
Dielectric Properties of Oligomers. VIII.

Table I. Boiling points of 2,4,6-heptanetriol triacetate

\begin{tabular}{lccc}
\hline Fraction & $\begin{array}{c}\text { Boiling point } /{ }^{\circ} \mathrm{C} \\
\text { (pressure } / \mathrm{Pa})\end{array}$ & Identification & wt $\%$ \\
\hline First fraction & $99-102$ & Syndiotactic & 23.1 \\
Second fraction & $(34.7)$ & Heterotactic & 49.3 \\
Third fraction & $92-94$ & & 27.6 \\
& $(32.0)$ & Isotactic & \\
$2,4,6-$ Heptanetriol $^{\mathrm{a}}$ & $90-94$ & & \\
\end{tabular}

a Non-fractionated mixture.

of Miyake and Kominami. ${ }^{9}$ The triol obtained was completely acetylated by a conventional procedure. $147 \mathrm{~g}$ of high-viscous 2,4,6-heptanetriol triacetate, a mixture of syndio-, hetero-, and iso-tactic stereoisomers, was obtained. Since the difference in boiling points among the three isomers was very small, it was too difficult to separate the mixture into pure isomers by conventional distillation. The isomeric mixture was therefore fractionally distilled (200 theoretical plates, Model 251, Perkin-Elmer) under reduced pressures. The boiling points of the collected fractions are given in Table I. Their purities were found to be above $99 \%$ by infrared (IR) spectra. Melting points were not determined, but the reported values are as follows: the syndiotactic, $32.5-33.5^{\circ} \mathrm{C}$; the heterotactic, $21-22^{\circ} \mathrm{C}$; the isotactic, glassy at room temperatures. ${ }^{10}$

\section{Dielectric Measurement}

A platinum concentric glass cell for liquid was immersed in a well-stirred alcohol-dry ice bath and the temperature was measured with a calibrated $\mathrm{Au}-\mathrm{Co}$ vs. $\mathrm{Cu}$ thermocouple. The dielectric constant and dielectric loss were measured in the pure liquid state by a ratio-arm transformer bridge (Type TR-1BK, Ando Electric Co.) over a frequency range from $23 \mathrm{~Hz}$ to $3 \mathrm{MHz}$. For this frequency range, it is not possible to measure the rotational relaxation of small molecules such as our isomers in the pure liquid state, since such small molecules relax very rapidly near room temperature. Our isomers except for the heterotactic one could be maintained in the "rotator phase" of the molecular dipoles by lowering the temperature slowly below the melting point. The heterotactic isomer did not remain in the liquid state long enough during measurement of the entire dispersion.

\section{Determination of Dipole Moments}

In order to eliminate concentration effects, the dipole moments of the three isomers in $\mathrm{CCl}_{4}$ were determined by the Halverstadt-Kumler method ${ }^{11}$ at $20.0^{\circ} \mathrm{C}$. Capacitance was measured with a Precision Capacitance Measurement System (type 1621, General Radio Co.). The minimum capacity reading was $10 \mathrm{aF}$. The capacitance cell (the vacuum capacity was $6.0645 \mathrm{pF}$ ) was a platinum concentric glass cell.

\section{RESULTS AND DISCUSSION}

\section{Static Dielectric Constant}

Large static dielectric constants are expected for 2,4,6-heptanetriol triacetate in the liquid state since this molecule has three polar side-groups in a short chain. The observed large static dielectric constants in Figure 1 show that the triacetates remain in the rotator phase throughout the measuring temperature range in that the dielectric constant for a solid composed of frozen dipoles is generally as small as 2-3. A plot of the static dielectric constant $v s$. reciprocals of absolute temperature is represented by a straight line in Figure 1, The marked difference in the static dielectric constants among the four samples can be explained by the difference in their dipole moments given in Table II. The large static dielectric constant in Figure 1 corresponds to the large dipole moment observed in the $\mathrm{CCl}_{4}$ solution. 
It is thus concluded that the molecular conformation of the isomers in $\mathrm{CCl}_{4}$ is not very different from that in the pure liquid state. The conformational analysis of the isomers in the pure liquid state was impossible.

Fujiwara et al. $^{7}$ determined the conformation of 2,4,6-heptanetriol triacetate in a $10 \mathrm{~mol} \%$ benzene solution by NMR. They concluded that a planar zig-zag may be the only possible conformation for the syndiotactic isomer, the isotactic trimer forms a helix, and that the spectrum of the heterotactic trimer can be interpreted in terms of a combination of isotatic and syndiotactic parts.

\section{Dielectric Relaxation}

In the case of a polymer chain, the principal

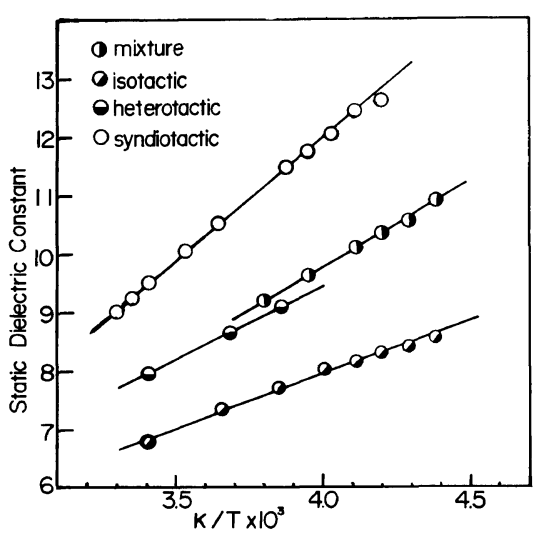

Figure 1. Temperature dependence of static dielectric constants of three stereoisomeric and non-fractionated 2,4,6-heptanetriol triacetate.

Table II. Dipole moments of 2,4,6-heptanetriol triacetate at $20.0^{\circ} \mathrm{C}$

\begin{tabular}{cccc}
\hline Isotactic & Heterotactic & Syndiotactic & Mixture \\
\hline $\begin{array}{c}9.9 \times 10^{-30} \mathrm{Cm} \\
(2.97 \mathrm{D})\end{array}$ & $\begin{array}{c}10.7 \times 10^{-30} \mathrm{Cm} \\
(3.21 \mathrm{D})\end{array}$ & $\begin{array}{c}11.5 \times 10^{-30} \mathrm{Cm} \\
(3.44 \mathrm{D})\end{array}$ & $\begin{array}{c}10.6^{-30} \mathrm{Cm} \\
(3.19 \mathrm{D})\end{array}$ \\
\hline
\end{tabular}

relaxation (the so-called " $\alpha$-relaxation") is generally associated with the micro-Brownian motion of chain-segments. This $\alpha$-relaxation often shows an asymmetric and wide distribution of relaxation times. In the case of a small molecule, on the other hand, the dielectric relaxation appearing in the lowfrequency range is mainly associated with rotation of the entire molecule. It is needless to say that the segmental motion of a polymer in the rubbery state is fundamentally different from the molecular rotation. However, Williams maintains that there is similarity between the relaxational behavior of a small molecule and that of an amorphous polymer. $^{12}$

The asymmetric Havriliak-Negami equation ${ }^{13}$ is very useful for analyzing various kinds of dielectric relaxations since this empirical equation includes the Cole-Cole and Davidson-Cole equations. The Havriliak-Negami equation is given as,

$$
\varepsilon^{*}-\varepsilon_{\infty}=\frac{\varepsilon_{0}-\varepsilon_{\infty}}{\left[1+\left(j \omega \tau_{0}\right)^{1-\alpha}\right]^{\beta}}
$$

where $\varepsilon^{*}$ is the complex dielectric constant, $\varepsilon_{0}$ and $\varepsilon_{\infty}$ are the limiting low- and high-frequency dielectric constants respectively, $j=\sqrt{-1}, \omega$ is the

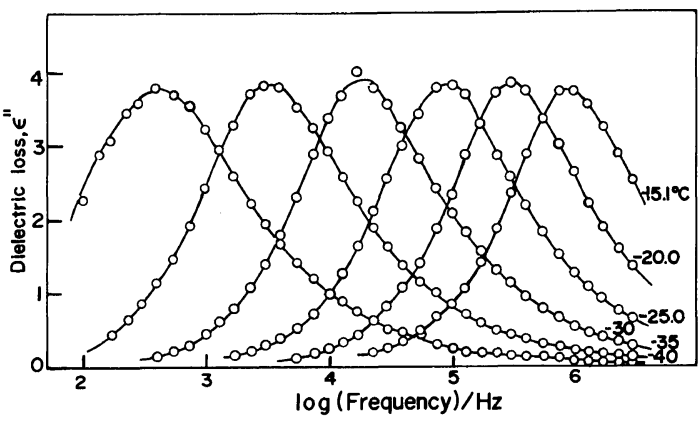

Figure 2. Dielectric loss curves of syndiotactic 2,4,6heptanetriol triacetate at various temperatures.

angular frequency, $\tau_{0}$ is the mean relaxation time, and $1-\alpha$ and $\beta$ are distribution parameters.

Figure 2 shows the frequency dependence of the dielectric loss of syndiotactic 2,4,6-heptanetriol triacetate at various temperatures. Mashimo and Chiba ${ }^{14}$ observed asymmetric loss curves for vinyltype polymers in dilute solutions. Their results show that asymmetric relaxation is associated with intramolecular motion in an isolate chain. In most cases, asymmetric relaxations were observed for chain molecules containing more than two polar 
groups. ${ }^{3,15,16}$ Several empirical equations as well as the Havriliak-Negami equation are proposed to describe asymmetric relaxations of polymers. ${ }^{17,18}$

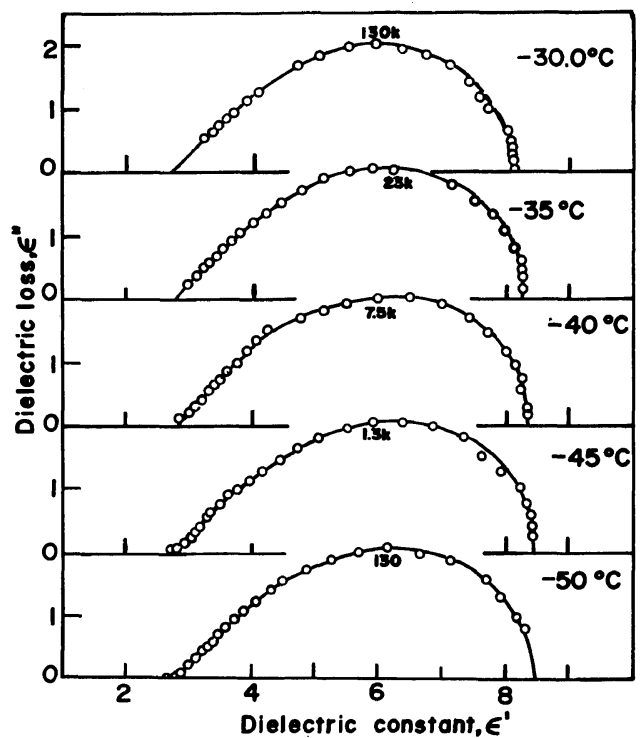

Figure 3. Cole-Cole plots of isotactic 2,4,6-heptanetriol triacetate at various temperatures. Numbers appearing with data points denote frequencies in $\mathrm{Hz}$.
Figure 3 shows the Cole-Cole plot for isotactic 2,4,6heptanetriol triacetate at various temperatures. The syndiotactic isomer also shows similar asymmetric arcs. From these arcs, the mean relaxation times $\tau_{0}$, the distribution parameters $1-\alpha$ and $\beta$ in the Havriliak-Negami equation were calculated by the method described in reference 13 . The numerical values of the experimental relaxation parameters are presented in Table III. The table indicates that the distribution parameter $1-\alpha$ is, in most cases, exactly or nearly equal to unity. These small values of $\alpha$ indicate the most striking characteristic of the chain molecules having plural acetate sidegroups. $^{4,5}$ When $1-\alpha$ is equal to unity, the Havriliak-Negami equation reduces to the Davidson-Cole equation, ${ }^{3}$

$$
\varepsilon^{*}-\varepsilon_{\infty}=\frac{\varepsilon_{0}-\varepsilon_{\infty}}{\left(1+j \omega \tau_{0}\right)^{\beta}}
$$

Since $\alpha \approx 0$, these asymmetric arcs of 2,4,6-heptanetriol triacetate can be also approximately represented by the Davidson-Cole equation.

\section{Distribution of Relaxation Times}

Depending on the distribution of relaxation

Table III. Limiting low- and high-frequency dielectric constants, mean relaxation times, and distribution parameters

\begin{tabular}{|c|c|c|c|c|c|c|}
\hline Isomer & Temp $/{ }^{\circ} \mathrm{C}$ & $\varepsilon_{0}$ & $\varepsilon_{\infty}$ & $\tau_{0} / \mathrm{s}$ & $1-\alpha$ & $\beta$ \\
\hline \multirow{6}{*}{ Syndiotactic } & -15.1 & 12.05 & 2.85 & $2.41 \times 10^{-7}$ & 1.00 & 0.64 \\
\hline & -20.0 & 11.80 & 2.70 & $7.58 \times 10^{-7}$ & 1.00 & 0.64 \\
\hline & -25.0 & 12.05 & 2.85 & $2.61 \times 10^{-6}$ & 1.00 & 0.64 \\
\hline & -30.0 & 12.45 & 2.85 & $1.22 \times 10^{-5}$ & 0.99 & 0.66 \\
\hline & -35.0 & 12.60 & 2.90 & $6.63 \times 10^{-5}$ & 0.98 & 0.65 \\
\hline & -40.0 & 13.00 & 2.90 & $5.31 \times 10^{-4}$ & 0.95 & 0.66 \\
\hline \multirow{5}{*}{ Isotactic } & -30.0 & 8.15 & 2.75 & $2.18 \times 10^{-6}$ & 0.98 & 0.56 \\
\hline & -35.0 & 8.30 & 2.80 & $8.38 \times 10^{-6}$ & 0.99 & 0.55 \\
\hline & -40.0 & 8.40 & 2.85 & $4.55 \times 10^{-5}$ & 0.97 & 0.58 \\
\hline & -45.0 & 8.50 & 2.85 & $2.34 \times 10^{-4}$ & 0.93 & 0.60 \\
\hline & -50.0 & 8.60 & 2.80 & $2.09 \times 10^{-3}$ & 0.95 & 0.55 \\
\hline Heterotactic & -20.0 & 7.40 & 2.35 & $4.68 \times 10^{-7}$ & 1.00 & 0.52 \\
\hline \multirow{4}{*}{ Mixture } & -30.0 & 10.10 & 3.02 & $3.84 \times 10^{-6}$ & 1.00 & 0.60 \\
\hline & -35.0 & 10.34 & 3.02 & $1.71 \times 10^{-5}$ & 1.00 & 0.59 \\
\hline & -40.0 & 10.55 & 3.00 & $7.58 \times 10^{-5}$ & 1.00 & 0.56 \\
\hline & -45.0 & 10.82 & 3.01 & $6.28 \times 10^{-4}$ & 0.98 & 0.57 \\
\hline Poly(vinyl acetate $)^{\mathrm{a}}$ & +70.0 & 8.65 & 3.20 & $2.24 \times 10^{-4}$ & 0.90 & 0.56 \\
\hline
\end{tabular}

a Calculated from the data of ref 2 . 
times, the dielectric relaxations of organic molecules in the liquid state have been classified into several types, e.g., Debye, Cole-Cole, Davidson-Cole, and Havriliak-Negami type. Each type must be associated with a particular relaxation mechanism, but it is very difficult to conclude definitely about what is responsible for each type of distribution. The origin of a distribution is related to a number of factors such as irregularity in the liquid structure, molecular-weight distribution, and superposition of consecutive relaxation mechanisms. As stated above, the chemical structure of the side-group in a chain molecule can be regarded as another important factor determining the distribution of relaxation times. For example, the distribution of relaxation times for 1,2,6-hexanetriol triacetate and glycerol triacetate are almost the same as that of the $\alpha$-relaxation of poly(vinyl acetate) ${ }^{4}$ This is also evident from the fact that the distribution of relaxation times for the isomers is the same as that of poly(vinyl acetate) as shown in Table III.

Therefore, as for the acetate chain molecules, we conclude from all these experimental results that the distribution of relaxation times is independent of

1) the skeletal chain-length,

2) the distribution of molecular weight,

3) the position and the number of the acetate groups in the chain

In order to investigate the isomeric effects on the distribution of relaxation times, the widths of the relaxation time distribution of syndiotactic, isotactic, and non-distilled 2,4,6-heptanetriol triacetate are compared in Figure 4, since it was expected that the coexistence of the isomers in a liquid must affect the relaxation behavior, especially, the width of the distribution of relaxation times. It is clear from Figure 4 that the distribution for the mixture is

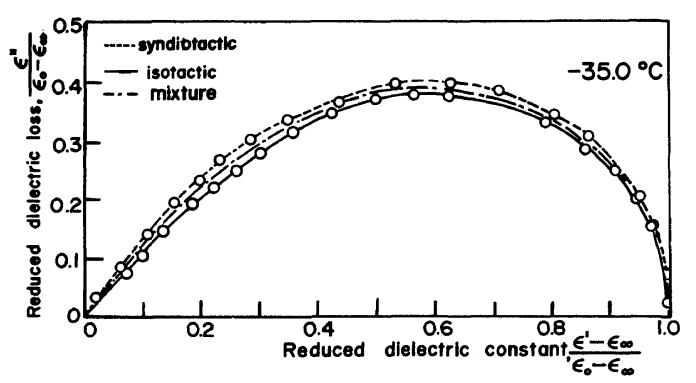

Figure 4. Comparison of the three arcs of syndiotactic, isotactic, and non-fractionated 2,4,6-heptanetriol triacetate on reduced Cole-Cole plots. unexpectedly close to those for the syndio- and isotactic molecules, although, in the three samples, the mixture has the largest entropy because it consists of three different molecules. The distribution of relaxation times for the mixture shows an intermediate values between those of the syndio- and isotactic isomer. The above observations lead to the conclusion that the main origin of the distribution of relaxation times characteristic of the acetate chain molecules may be the cooperative motion of the main chain and the side-chain. The asymmetry of the distribution is also independent of the coexistence of different isomers. Glarum ${ }^{19}$ considers that the dipole relaxation in the skewed arc relaxation occurs as a result of a combination of two mechanisms such as "holes" in the lattice defects and dipolar orientation.

\section{Relaxation Times}

The calculated mean relaxation times are tabulated in Table III and plotted against the reciprocal absolute temperature in Figure 5. As seen in this figure, the difference in the relaxation times between syndio- and iso-tactic heptanetriol triacetate is comparatively large in spite of having the same molecular weight. The relaxation times for the mixture also show intermediate values between the syndioand the iso-tactic molecules. The relaxation time for molecular rotation in the liquid state depends on two factors: the macroscopic viscosity of the medium and the molecular radius of rotation. ${ }^{20}$ The

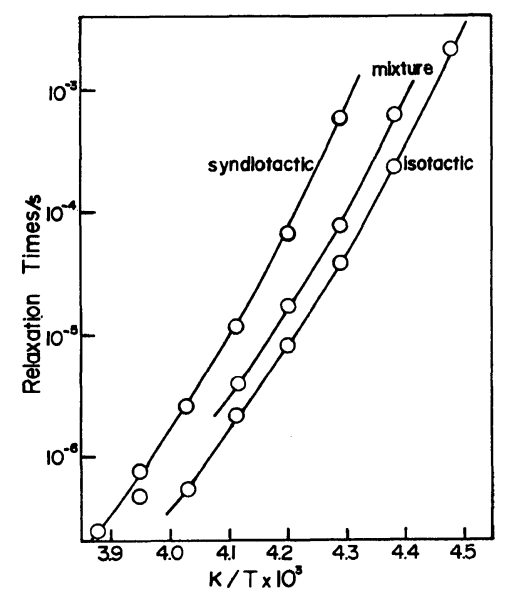

Figure 5. Temperature dependence of relaxation times of syndiotactic, isotactic, and non-fractionated 2,4,6heptanetriol triacetate. 
dipole-dipole force, which depends on the value of the dipole moment, is larger for the syndiotactic isomer $(3.44 \mathrm{D})$ than for the isotactic isomer $(2.97$ D). This larger molecular interaction leads to a larger viscosity of the medium, which accordingly contributes to longer relaxation times of the syndiotactic isomer. Another reason for the longer relaxation times of the syndiotactic isomer seems to be the larger molecular radius of rotation of this molecule, since it is considered that the average molecular radius of this isomer, which is apt to form a helical structure, is larger than that of the isotactic isomer having a planar zig-zag structure. Anyway, these isomers must have sphere-like structure because they each have the three large side-groups in the short chains.

The Arrhenius plots of the relaxation times are not represented by straight lines but by curves. Such non-linear relationships are seen not only in rubbery polymers, but also in hydrogen-bonded and glassy liquids, ${ }^{4}$ in which in addition to the variation in motional energy another temperature-dependent factor such as the number of hydrogen bonds or free volume shows such a non-linear behavior. Powles ${ }^{21}$ plotted relaxation time against reciprocal absolute temperature for a variety of liquids and noted that the departure of the plots from straight lines is large for viscous supercooled liquids but small for lowviscous liquids.

\section{REFERENCES}

1. D. J. Mead and R. M. Fuoss, J. Am. Chem. Soc., 63, 2832 (1941)
2. Y. Ishida, M. Matsuo, and K. Yamafuji, Kolloid Z. Z. Polym., 180, 108 (1962).

3. D. W. Davidson and R. H. Cole, J. Chem. Phys., 19 , 1484 (1951).

4. E. Ikada and T. Watanabe, J. Phys. Chem., 78, 1078 (1974).

5. E. Ikada, T. Sugimura, T. Aoyama, and T. Watanabe, Polymer, 16, 101 (1975).

6. E. Ikada, T. Sugimura, and T. Watanabe, J. Polym. Sci., Polym. Phys. Ed., 16, 907 (1978).

7. Y. Fujiwara, S. Fujiwara, and K. Fujii, J. Polym. Sci., A-1, 4, 257 (1966).

8. D. Daskocilova, J. Stokr, E. Votavova, B. Schneider, and D. Lim, J. Polym. Sci., C, No. 16, 2225 (1967).

9. Y. Miyake and T. Kominami, Kogyo Kagaku Zasshi (J. Chem. Soc. Japan, Ind. Chem. Sect.), 60, 1340 (1957).

10. D. Lim, E. Votavova, J. Stokr, and J. Petranek, J. Polym. Sci., B, 4, 581 (1966).

11. I. F. Halverstadt and W. D. Kumler, J. Am. Chem. Soc., 64, 2988 (1942).

12. G. Williams and P. H. Hains, Faraday Symp. Chem. Soc., No. 6, 14 (1972).

13. S. Havriliak and S. Negami, J. Polym. Sci., C, No. 14, 99 (1966).

14. S. Mashimo and A. Chiba, Polym. J., 5, 41 (1973).

15. S. K. Garg, W. S. Lovell, C. J. Clemett, and C. P. Smyth, J. Phys. Chem., 77, 232 (1973).

16. E. Ikada and T. Watanabe, J. Polym. Sci., Polym. Chem. Ed., 10, 3457 (1972).

17. G. Williams and D. C. Watts, Trans. Faraday Soc., 66, 80 (1970).

18. A. K. Johnsher, Colloid Polym. Sci., 253, 231 (1975).

19. S. H. Glarum, J. Chem. Phys., 33, 639 (1960).

20. See, e.g., C. P. Smyth, "Dielectric Behavior and Structure," McGraw-Hill Book Co., New York, N.Y., 1955, Chapter IV.

21. J. G. Powles, "Molecular Relaxation Processes," Chemical Society Special Publication, No. 20, Academic Press, London, 1966, p 131. 